Cinco, después de Babel (un lugar para la traducción y para la tradicción)

\title{
La relectura contemporánea de un clásico italiano: Leopardi en las publicaciones periódicas catalanas después de la Gran Guerra
}

\section{Camps, Assumpta}

Assumpta Camps Sobre la autora Universidad de Barcelona, España

El hilo de la fábula

Universidad Nacional del Litoral, Argentina

ISSN: $1667-7900$

ISSN-e: $2362-5651$

Periodicidad: Anual

vol. 19, núm. 21,2021

revistaelhilodelafabula@fhuc.unl.edu.ar

Recepción: 14 Noviembre 2020

Aprobación: 17 Enero 2021

DOI: https://doi.org/10.14409/hf.v0i21.10569
Resumen: En el presente artículo estudiaremos un capítulo de la recepción de los Canti de G. Leopardi en España en la primera mitad del siglo XX, en concreto, las traducciones al catalán que se publicaron en la prensa periódica en los años comprendidos entre la Gran Guerra y la Guerra Civil española. Para ello, nos centraremos en el análisis detallado de las traducciones realizadas por J.M ${ }^{a}$ Garganta para «La Nova Revista», con el fin de ver la lectura que nos ofrece de Leopardi.

Palabras clave: Historia de la Traducción, Recepción Literatura Italiana, G Leopardi, JMª Garganta.

\begin{abstract}
In this paper a chapter on the reception of the Canti by G. Leopardi in Spain in the first half of the 20th century will be analized, specifically, the translations into Catalan that were published in the journals and newspapers in the years between the I World War and the Spanish Civil War. The text will be focused on the detailed analysis of the translations made by J.Ma Garganta for «La Nova Revista», in order to see his reading of Leopardi.
\end{abstract}

Keywords: History of the Translation, Reception of Italian Literature, G Leopardi, JMa Garganta.

La recepción de Giacomo Leopardi en la Península, a diferencia de la de Alessandro Manzoni, resulta algo tardía en el tiempo, y desde luego no es coetánea. Sus inicios deben situarse hacia mediados de la década de los 70 del siglo XIX. Se desarrolla ampliamente con el pasar de los años, enriqueciéndose con múltiples traducciones y versiones, hasta dar lugar a una historia de la traducción singularmente rica, no solo en español, sino también en otras lenguas peninsulares, como por ejemplo el catalán. Una historia rica, sin duda, pero muy orientada a los Canti, de un modo casi exclusivo.

En la historia de la traducción en catalán de Leopardi los Canti contaron ya a finales del siglo XIX con una serie de versiones que el mallorquín Joan Lluís Estelrich recogió en su muy célebre Antología de poetas líricos italianos, obra publicada en Palma de Mallorca en 1889. En ella nos ofreció varias de las primeras traducciones de Leopardi, como una versión catalana de «Imitazione» titulada «La fulla», obra de M. Obrador i Bennassar. Junto a ella, la de «La sera del dì di festa» («La nit del dia de festa») que llevó a cabo Joan Sardà, responsable 
asimismo de la traducción de «Aspasia» que apareció recogida en uno de los apéndices de dicha antología.

Sin duda fue Alfons Maseras quien más se ocupó de Leopardi en los círculos literarios catalanes a principios del siglo XX. Su versión íntegra al catalán de los Canti, publicada por la editorial Oasis de Barcelona, se remonta a la época de la guerra civil española, pues se dio a conocer en $1938^{1}$ (un año después del centenario de la muerte del autor de Recanati, para el que Maseras ya preparó en su día el opúsculo titulado Primer Centenari de Giacomo Leopardi 1798-1837, ${ }^{2}$ que contenía en versión bilingüe ítalo-catalana los cantos «A Silvia» ${ }^{3}$ e «Il sabato del villaggio $»$ - poema que tituló, por cierto, «El disabte del poblet $\gg)$. Poco antes, en 1935 para ser exactos, Maseras ya había publicado en uno de los principales periódicos generalistas de Barcelona, «La Veu de Catalunya», alguna pequeña muestra de lo que habría de ser su ingente labor de difusión de la poesía leopardiana entre nosotros. Nos referimos a su versión al catalán del «Frammento XXXIX» de los Canti, que él dio a conocer simplemente como «Poema XXXIX», en agosto de ese año $1935 .{ }^{4}$ Pero su dedicación a Leopardi venía de lejos. De hecho, muchos años antes ya había publicado su traducción del canto «L'infinito» («L'infinit») en la revista Catalunya Artística, en concreto en 1901.

A partir de la Gran Guerra abundan las traducciones al catalán de los poemas de Leopardi, en su mayoría realizadas por escritores de las Islas Baleares. Aparecen publicadas esporádicamente, de un modo bastante disperso, en las diferentes publicaciones periódicas catalanas de la época (también de la ciudad de Barcelona), incluidos los periódicos generalistas (característica que era muy habitual en ese momento, aunque puede sorprender hoy en día, tanto al lector de poesía como al de periódicos). En este sentido, recordaremos aquí, por ejemplo, las que se dieron a conocer en La Revista de Barcelona. A saber: «La nit del dia de festa» de Miquel Ferrà (10 de junio de 1915), ${ }^{5}$ que el mallorquín incluyó más tarde en su volumen de 1920 titulado Les muses amigues (XVI traduccions en vers), págs. 33-34, publicado por la Estampa de Marquès i Mayol de Palma de Mallorca. En el mismo orden de cosas, «El dissabte de la vila» (traducción de «Il sabato del villaggio), obra de Miquel Forteza, ${ }^{6}$ publicada en 1917 en La Revista (págs. 151-152); como también, en julio de 1922, «La vida solitària» de Tomàs Garcès; y, unos años más tarde, en 1928 para ser exactos, la que llevó a cabo Ricard Permanyer, titulada «Les recordances» (traducción de «Le ricordanze»), págs. 75-80.

Por su parte, el periódico La Veu de Mallorca publicó por la misma época otras traducciones al catalán de los Canti leopardianos. En concreto, en 1918, la traducción de «La sera del dì di festa» que realizó Miquel Ferrà (que ya hemos citado arriba), así como otra de «L'infinito» («L'infinit») del también mallorquín Miquel Forteza. Joan Estelrich menciona ${ }^{7}$ que este último dio a conocer asimismo en La Veu de Mallorca una traducción al catalán del idilio «A Silvia» en 1922, y que dicho periódico mallorquín publicó en julio de 1923 una traducción al catalán de «A la Itàlia» (traducción de «All'Italia»), obra de Guillem Colom. ${ }^{8}$ Sin embargo, parece muy improbable que así fuera, pues La Veu de Mallorca permaneció clausurada desde marzo de 1919 hasta enero de 1931, según hemos podido comprobar. 
Tres traducciones más de Leopardi, esta vez realizadas por Agustí Esclasans, se remontan también a estos años. Se trata de «La calma desprès de la tempesta» (traducción de «La quiete dopo la tempesta», que se publicó en La Nau de Barcelona en 1917), así como «Fragments» (traducción de «Frammento XXXIX»), que presentó La Nova Revista en 1920 (págs. 215-217), y unos años más tarde, ya en octubre de 1928, el «Darrer cant de Safo» (traducción de «Ultimo canto di Safo»).

Joan Estelrich contribuyó a la difusión de un buen número de traducciones de Leopardi realizadas por escritores mallorquines con la publicación de su conferencia sobre el autor de Recanati, titulada Entre la vida i els llibres (La Veu de Mallorca, 1918, parcialmente), recogida más tarde en su volumen Assaigs. ${ }^{9}$ Estelrich menciona, en concreto: «A la Itàlia», de Guillem Colom (a la que ya hemos aludido antes); «L'Infinit», «A Sivia» $\mathrm{y} \ll$ El dissabte de la vila», de Miquel Forteza (también citadas anteriormente); ${ }^{10} \ll$ A la lluna», de Cristòfol Magraner (publicada también en La Veu de Catalunya el 10 de mayo de 1918); y «La nit del dia de festa», de Miquel Ferrà (que ya hemos reseñado). Todas ellas iban inicialmente acompañadas por una versión catalana de «Le ricordanze», obra de Joan Pons (también de 1918), que permaneció inédita hasta entonces.

El año 1918 conoció además la versión del «Ultimo canto di Safo» («Darrer cant de Safo») que realizó Agustí Esclasans, así como la de Ricard Permanyer de «Le ricordanze» («Les recordances»), ambas ya mencionadas arriba, como también varias traducciones dispersas de Leopardi, entre las que recordaremos una de «A se stesso», obra de Josep $\mathrm{M}^{\text {a }}$. López-Picó (traducida como «De Leopardi a ell mateix») -versión que también se recogió en el volumen de este autor, titulado Temes. Exercicis de geografia lírica (Barcelona 1928) -, y dos más de Josep $M^{a}$. de Garganta: «L'infinit»y «Fragment» (traducción, esta vez, del «Frammento XXXVIII» de Leopardi), que fueron presentadas en la revista como Poemes de Leopardi (en número 16 de La Nova Revista, de abril de 1928).

Ahora bien, en esta historia de la traducción catalana de los Canti destacan muy especialmente, además de la versión íntegra de Maseras que ya mencionamos, las que hiciera el escritor Josep Carner, publicadas todas ellas entre el mes de febrero de 1922 y junio de 1924 en un periódico barcelonés generalista de gran difusión, como era por entonces La Veu de Catalunya. Por el gran número de las mismas, la naturaleza de estas, y la centralidad de la figura de Carner en las letras catalanas de los años anteriores a la guerra civil española, el análisis en profundidad de estas traducciones merece, sin lugar a dudas, un capítulo aparte.

\section{El Leopardi de Josep Ma de Garganta en La Nova Revista}

El mismo año 1928 en que se dieron a conocer otras poesías de Leopardi en traducción catalana, el escritor Josep $\mathrm{M}^{\mathrm{a}}$ de Garganta publicó en La Nova Revista sus versiones de dos de los Canti leopardianos: el celebérrimo idilio «L'infinito» y el «Frammento XXXVIII», que Garganta nos presentará tan solo como «Fragment». ${ }^{11}$

De Garganta, nacido en 1878 en un pueblecito de la comarca gerundense de la Garrocha, en el pre-Pirineo catalán, era poco más que un autor de comarcas, escasamente conocido en los ambientes literarios de Barcelona de principios del 
siglo XX. Y lo era aún en menor medida debido a los problemas de salud que le tuvieron apartado entre 1901 y 1908. Sin embargo, fue un innegable animador de la vida cultural de Olot (la capital de dicha comarca) desde 1909 y hasta su muerte, participando en los Juegos Florales de dicha ciudad, en el aniversario del certamen Literario-artístico de 1909, o bien organizando, en 1910, lo que sería su Museo-Biblioteca. No es precisamente hasta 1910 cuando publica su primer poemario, titulado Arquimesa, al que habría de seguir, en 1912, Evocacions, que fue, por cierto, muy admirado por el también poeta Miquel Costa i Llobera, una figura literaria de primer orden en los ambientes catalanes de la época.

Destaca, en esos años, la labor de J.M ${ }^{\text {a }}$ de Garganta como traductor (la cual alternó con su propia producción literaria), ya fuera del italiano, como del inglés, el portugués, el francés, el rumano y también el latín. Dichas traducciones fueron, años más tarde, recogidas en parte por su amigo Josep $\mathrm{M}^{\mathrm{a}}$ Capdevila en Poesies de Josep $M^{a}$ de Garganta, un volumen que se editó en 1968. Pero inicialmente se dieron a conocer en varias revistas y periódicos de la época, como sucedió con sus traducciones de Leopardi. Entre los autores que tradujo al catalán, además del poeta de Recanati, se hallan Matteo Boiardo, Alessandro Manzoni, Joachim du Bellay, Ronsard, Wordsworth, Francis Jammes, Charles L. de Chénedollé, Félix Arvers, Alfred de Vigny, J.Ma . de Heredia, Gustave Nadaud, Vasile Alecsandri, Thomas Gray, Tennyson, Wordsworth y, especialmente, H.W. Longfellow de quien empezó a traducir el poema Evangelina que el periódico El Deber publicó en forma de suplemento.

A decir verdad, la imagen de Leopardi difundida desde las páginas de La Nueva Revista no solo se construyó sobre la base de dichas traducciones al catalán, como sabemos. El interés en esta publicación por el poeta italiano muestra, más allá de la preocupación por invocar y traducir a uno de los clásicos universales de la época moderna, una cierta persistencia de un gusto romántico que se hallaba ya hasta cierto punto periclitado. Esto se pondrá también de manifiesto en algunas otras colaboraciones críticas de esta publicación, como las «Reflexions sobre el sentimentalisme» de Jordi Arqué, publicadas en marzo de $1929,{ }^{12}$ en las que Leopardi se nos equipara a Foscolo y a Lamartine en su vivir atormentado. Así podemos leerlo en el siguiente fragmento:

[...] Leopardi, el líric italià de més força del seu temps, pesimista que vessà en les seves obres el dolor físic del seu viure malaltís i turmentat. (Arqué, 1929:191). ${ }^{13}$

Estas opiniones, que insisten, como vemos, en la imagen de un Leopardi pesimista, enfermizo y atormentado, prevalecen en esta publicación en esos años, alimentando un tópico que era muy común en la época. A esta imagen del autor se incorporan, en parte por contraste, las dos traducciones de J. $\mathrm{M}^{\mathrm{a}}$ de Garganta que veremos aquí: «L'Infinit» (307) y «Fragment» (308).

\section{$\ll$ L'Infinit» («L'Infinito»)}

Compuesto en Recanati en 1819, «L'Infinito» es, como sabemos, un idilio de 15 versos en endecasílabos sciolti, que nuestro traductor lo convierte en un poema de 16 versos, en los que se mantiene el metro y se prescinde completamente de la rima. La traducción, en su conjunto, presenta ciertas interpretaciones libres, a menudo próximas a una complacencia por el calco italianizante, así como la 
supresión de algunos rasgos estilísticos importantes del autor de Recanati, como veremos.

Se abre con una inversión en el v. 1 que quita énfasis al adjetivo «car» que emplea de Garganta: «car», aunque pueda parecer un calco italianizante, corresponde en realidad a una de las acepciones de este término en catalán normativo («caro $\mathrm{mi}$ fu» $>$ «em fou cara»). Lo mismo sucede a propósito de «ermo» [es decir, «solitario»] > «erma». Junto a estas propuestas, una opción de traducción claramente errónea a nuestro entender, y que desvía la atención del lector: la que hace referencia al traslado «colle» > «collada», que, aunque recuerda el término italiano tan propio del universo leopardiano («colle»), con el que el autor se refiere al Monte Tabor, tiene de hecho un significado significativamente diferente. La supresión de la coma (v. 1) anterior a la conjunción copulativa se sucederá en otras ocasiones a lo largo de esta traducción, y no comporta una alteración significativa en el texto.

En los vv. 2-3, de Garganta someterá el fragmento a una interpretación que en ocasiones da como resultado una traducción amplificadora de carácter explicativo (por ejemplo: «siepe» > «tanca frontera»), de todo punto innecesaria a nuestro modo de ver, mientras que en otros casos deriva de una mala lectura o dificultad de comprensión del original. Así ocurre, por ejemplo, a propósito del latinismo «ultimo», que Leopardi utiliza con el significado de «estremo»; contrariamente a cuanto se puede ver con relación al verbo «mi fingo» [es decir, «me imagino»] > «m'imagino» en el v. 7). Por su parte, la reescritura de estos versos tiene como consecuencia una reordenaci en el v. , me imagino] $>2$ m'ntece con relaciinaltantes. la enaci una de las acepciones del $t$, en las que rompoe el encadenamienón de los elementos constitutivos de la frase que se llevada a cabo de modo quiasmático ( «che da tanta parte / dell'ultimo orizzonte il guardo esclude». > «que a ma vista / de l'ultim horitzó gran part oculta»).

En los vv. 4-7 se observan dos supresiones importantes. En efecto, la reordenación de estos versos comporta la eliminación de dos enjambements muy relevantes en el poema («interminati / spazi» y «sovrumani / silenzi»), solo en parte compensados por el que de Garganta introducirá entre los vv. 5-6 («calma / profunda»), enjambement que destaca en particular en la traducción este elemento de la calma frente a los demás («espacios infinitos», «silencios sobrehumanos»). Asimismo, hay que mencionar la desaparición de las tres conjunciones copulativas, que tienen un efecto muy marcado en el original. Por otra parte, el añadido de «me plau fantasiar» corresponde a una pura invención del traductor (aunque acorde con en el contexto, si queremos), que duplica, hasta cierto punto, la noción transmitida con el verbo «mi fingo», correctamente traducido como «m'imagino» en el v. 7 . El cambio en la puntuación que se produce en dicho v. 7 (pasamos del punto y coma, a la coma) elimina una división importante en el poema, indicativa de un importante cambio de sensación, que pasa de la calma al terror. A diferencia de cuanto hemos visto anteriormente, de Garganta decidirá conservar el enjambement en los vv. 7-8, si bien los reescriba. De un modo tal que cambia sutilmente el punto de vista del original, centrando la atención no ya en el corazón (como hiciera Leopardi), sino en la calma (término que queda implícito), la cual suscita terror («ove per poco / il cor non si spaura.» $>$ «tal, que em dóna [la calma] / una ombra de terror»). 
Por su parte, los vv. 8-11 se ven igualmente reescritos. En la reordenación de sus elementos, se suprimirá nuevamente un enjambement importante («quello / infinito silenzio»), al tiempo que de Garganta invertirá los dos elementos de la comparación - que presentan un contraste entre opuestos-: «silenci»«veus». De modo tal que ya no es que el elemento natural contribuya a la definición de ese «infinito silencio» que adquiere un valor metafórico, como ocurre en el idilio leopardiano, sino que la comparación se produce exactamente al revés. Pero, sobre todo, de Garganta interpretará muy libremente el verbo «stormir» > «arremorar» [es decir, «avalotar»]. Y decimos muy libremente porque mientras Leopardi se centra en el viento que agita levemente las hojas produciendo un ruido suave, como un ligero crujir, la escena que nos transmite el traductor es completamente distinta: la de las hojas agitadas violentamente por el viento (sin especificar nada con respecto al sonido, aunque se puede inferir quizá que un ligero crujir apunta a un ruido algo más intenso, acorde con la violencia del movimiento). Por esta vía observamos que de Garganta altera substancialmente uno de los términos de la comparación («voce», al plural en la traducción catalana: «veus»), procedente del entorno natural, mediante el cual el autor nos describe ese «infinito silencio» que se menciona en el idilio.

No siempre la interpretación libre es fuente de problemas en esta traducción, a decir verdad. En ocasiones, ocurre lo contrario: la fuerte adherencia al original evidencia, en realidad, una incomprensión de base. Es lo que ocurre, por ejemplo, en los vv. 11-13, que se nos presentan fuertemente dislocados en el original leopardiano. Destaca aquí la traducción «stagioni» [= «età», en Leopardi] > «estacions» (nótese, además, que se utiliza el plural), que se halla absolutamente fuera de lugar, y ante cuya incomodidad probablemente de Garganta siente la necesidad de introducir una preposición de relativo, acorde con su lectura del texto («que ja finiren»).

En la parte final del poema, de Garganta reescribe los versos originales de manera por momentos amplificadora, con el resultado de añadir un verso más al idilio leopardiano (como ya se dijo arriba). Destacan, en este sentido, por un lado, el añadido innecesario de «en mig de les onades». Por el otro, la interpretación implícita que hallamos en «s'annega» > «s'enfonsa» (que evita, por un lado, la connotación de muerte por ahogamiento, y por la otra, sobre todo la noción de perderse, de abandonarse, a la que Leopardi alude claramente en su poema). Y, por último, la opción por un término de carácter poético, como es «pèlag», en lugar de «mar» (sin duda por motivos de métrica, en primer lugar). Pero destaca también, y muy especialmente, la supresión del fuerte enjambement existente entre los vv. 13-14 («questa / immensità»), y la eliminación de los dos puntos del final del v. 14, que conferían al último verso del idilio un carácter claramente conclusivo. Al suprimir ambos rasgos, la traducción desvirtúa fuertemente el original en este punto.

\section{«Fragment» («Frammento XXXVIII»)}

En lo concerniente al «Frammento XXXVIII», compuesto en Recanati 1818, la traducción de Garganta se verá recogida simplemente, y sin presentación de ningún tipo, bajo un título simplificador como es «Fragment», y publicada a continuación de la anterior en la misma revista. El original presenta, como 
sabemos, una forma algo más compleja. En efecto, los 15 versos se distribuyen en tercetos de endecasílabos con rima encadenada. De Garganta conservará la métrica original, y también su distribución estrófica, pero se mostrará bastante más laxo en cuanto a la rima, que (a diferencia de cuanto vimos anteriormente) en este caso intenta mantener en lo posible, salvo por algunas ocasiones en las que rompe el encadenamiento que esta tenía en el original.

La traducción se abre con la reescritura de los vv. 1-3 en los que de Garganta lleva a cabo varias transformaciones. Por una parte, la eliminación del pronombre personal («io») y del adverbio («qui») que nos ubicaban inmediatamente en este canto leopardiano, a diferencia de cuanto ocurre en la traducción. Por el otro, la interpretación de «intorno» > «entorn», que cae en el calco lingüístico (si no se utiliza la locución adverbial «a l'entorn»). Contrariamente al acierto que observamos en el traslado de «limitare» $>$ «llindar», e incluso en el de la construcción con valor final «acciò che» (= «accioché») > «perquè», indicativa sin duda de un registro áulico en el poema original. Y, por último, el añadido de «amada», que explicita, interpretándolo, el pronombre personal del v. 3 ( «la ritenga», la cursiva es mía).

En los vv. 4-6, la traducción cae de nuevo en la interpretación libre, no solo a propósito del adverbio «pure» (que Leopardi emplea al inicio del v. 4 con valor de «anche»), trasladado sin relación alguna como «bé», sino también en cuanto a la imagen tópica de la aurora que se halla en el v. 5, en su despertar de nuevo cada día («l'autora in ciel ridesta»), imagen que se pierde en la traducción. De Garganta, optará por una solución translaticia curiosa en este punto ( «l'albor xalesta»), que por un lado, se aleja notablemente del significado original (pues «albor» es, de hecho, un pez de agua dulce, y «xalesta» equivale a «divertida»), al tiempo que lo recuerda vagamente en el plano fónico. Por otra parte, decide eliminar el adjetivo «errante» (con lo que se pierden algunos matices importantes del poema), así como el énfasis sobre el verbo «muggire» (que se repetía en los vv. 4 y 5), el cual traduce acertadamente por «bramulava». Aflora un nuevo calco italianizante en el uso del substantivo «floresta» (como traducción de «foresta»), el cual no existe en catalán normativo como tal; a diferencia de cuanto sucede, en cambio, con relación al adjetivo «care» del v. 7 .

De hecho, los vv. 7-9 se nos presentan notablemente transformados en esta traducción. De Garganta reescribe y altera (con el añadido de la ruptura que comporta aquí el punto y coma) el fuerte efecto acumulativo de los varios vocativos con los que el autor invoca a los elementos naturales. No solo eso, también transforma una parte de ellos («o piante»), al tiempo que añade otras referencias de su propia invención, y recompone las exclamativas del original, incrementando su número. Cabe mencionar aquí el traslado de una expresión de raigambre en la lírica italiana como es «donna mia», que se ve actualizada -y también algo banalizada- como «amada». El resultado es bastante poco satisfactorio en su conjunto. Destaca, en este sentido, especialmente la duplicación del objeto directo con la partícula «en» del v. 8. Pero también las invenciones y supresiones que desvirtúan el poema leopardiano, ya sea en su plano semántico como en sus rasgos estilísticos ( «O care nubi, o cielo, o terra, o piante, / parte la donna mia: pietà, se trova / pietà nel mondo un infelice amante. $\gg\rangle\langle$ Oh 
cel, oh terra; bromes, pietat! / Se'n va l'amada! Pietat, si en trova / enlloc del món l'amant desventurat.»).

En el terceto siguiente, de Garganta lleva a cabo una nueva reescritura de los versos originales (vv. 10-12), reordenando los elementos de la frase y anteponiendo el imperativo al vocativo (v. 10). El cambio en la puntuación (pasamos de una coma a un punto y coma), añade una nueva pausa que separa los dos verbos recogidos en este verso («ti sveglia»y «fate»), al tiempo que introduce una ruptura en el mismo. Por otra parte, de Garganta traducirá el primer vocativo recurriendo sorprendentemente a un castellanismo que rebaja el tono poético («turbine» > «torbellí»), e interpretará libremente el segundo de ellos («nembi») como «tempesta», al tiempo que introduce lo que podríamos considerar una sinécdoque en el traslado de «il dì > «sa claror», desplazando el acento sobre la luz.

La traducción concluye con una terceto igualmente bastante interpretativo en el que nuestro traductor opta de nuevo por la reescritura del verso. Por ejemplo, el v. 14, que de Garganta traduce del siguiente modo: «posan [es decir, reposan] l'erbe e le frondi» > «la remor / de les fruites s'aquieta». Como se comprobará, no solo ha llevado a cabo una inversión en los elementos de la frase, sino que opera una interpretación muy libre en cuanto a «frondi», e introduce una connotación de ruido ajena por completo al original («remor»), así como un enjambement. En otros momentos, sin embargo, la solución propuesta resulta más acertada, como sucede en «le luci» > «els ulls», o bien «crudo Sol» > «sol cruel», si bien no deja de alterar el texto, ya sea porque suprime la mayúscula que personificaba al «sol», o porque elimina la función de epíteto del adjetivo empleado. La reescritura de estos versos conduce, de hecho, a la supresión de la fuerte dislocación que hallamos en el original (sobre todo mediante la separación del substantivo y el adjetivo que le corresponde), dislocación con la cual Leopardi quiere cerrar este canto dramáticamente: «e m'abbarbaglia / le luci il crudo Sol pregne di pianto». [literalmente: «i m'enlluerna els ulls el cruel Sol banyats de plor».] > «i enlluerna / el sol cruel mos ulls banyats de plor». De un modo tal que la solución de traducción que de Garganta nos propone cumple a la vez la función de reordenar y explicitar el original, aunque sea a costa de la pérdida de énfasis y de carga emotiva de este, un rasgo que ya hemos ido viendo recurrentemente en estas traducciones.

En conclusión, la lectura que J. $\mathrm{M}^{a}$ Garganta nos ofrece de Leopardi a través de sus traducciones publicadas en 1928 es bastante libre e interpretativa, afectando al plano semántico de los poemas, así como a la métrica y a rasgos estilísticos importantes de los originales leopardianos, que se ven así en parte banalizados.

\section{Referencias}

ARQUÉ, Jordi (1929). Reflexions sobre el sentimentalisme. La Nova Revista, VII(27), págs. 184-198.

ESTELRICH, Joan (1926). Entre la vida i els llibres. En Assaigs, tomo IV. Llibreria Catalònia.

FORTEZA, Miquel. (1997). Poemes i traduccions. Barcelona.

LEOPARDI, Giacomo. (1928). Poemes de Leopardi: 'L'Infinit', 'Fragment' (trad. Josep $\mathrm{M}^{a}$ de Garganta). La Nova Revista, IV(16), págs. 307-308. 
LEOPARDI, Giacomo. (1935). «Poema XXXIX» (trad. A. Maseras). La Veu de Catalunya.

LEOPARDI, Giacomo. (1938). Cants (tradu. Alfons Maseras). Oasis.

MASERAS, Alfons. (1937). Primer Centenari de Giacomo Leopardi 1798-1837. Casal de Cultura, 1.

\section{Notas}

1 Cfr. LEOPARDI, Giacomo (1938). Cants, traducción de Alfons Maseras. Barcelona: Oasis.

2 MASERAS, Alfons. (1937). Primer Centenari de Giacomo Leopardi 1798-1837. Casal de Cultura, (1).

3 Esta traducción se había dado a conocer inicialmente en la revista Catalunya de Buenos Aires, en diciembre de 1936, p. 50.

4 LEOPARDI, Giacomo. (1935). «Poema XXXIX» (trad. de A. Maseras). La Veu de Catalunya, 4-VIII-1935.

5 Más tarde recogida también en otro periódico generalista, La Veu de Mallorca, en 1918, y posteriormente en el volumen de 1920 que hemos mencionado.

6 Las traducciones de Miquel Forteza se publicaron en época más reciente en FORTEZA, Miquel (1997). Poemes i traduccions.

7 Cfr. ESTELRICH, Joan. (1926). Entre la vida i els llibres, en: Assaigs, tomo IV, Barcelona: Llibreria Catalònia. No confundir con J.Ll. Estelrich, autor de la célebre Antología de poetas líricos italianos (1889), también mallorquín.

8 Por lo que sabemos, la fecha de composición de esta traducción fue, como mínimo, 1918 , aunque no nos ha sido posible localizarla por el momento, ni verificar este dato. Por las razones que exponemos aquí, se comprende que no hemos podido encontrar esta traducción, pues está citada recurrentemente con una fecha que es imposible. Por lo mismo, no se analizará aquí, a la espera de que se puede comprobar este dato.

9 ESTELRICH, Joan. (1926), op. cit., pp. 18 y ss.

10 Sin embargo, no hemos podido verificar la existencia de esta traducción al catalán de «A Silvia», que según la crítica apareció en La Veu de Mallorca en 1922, fecha harto improbable, ya que por entonces esta publicación estaba clausurada.

11 LEOPARDI, Giacomo. (1928). Poemes de Leopardi: 'L'Infinit', 'Fragment' (trad. de Josep Ma de Garganta). La Nova Revista, IV(16), 307-308.

12 ARQUÉ, Jordi. (1929). Reflexions sobre el sentimentalisme. La Nova Revista, VII(27), págs. 184-198.

$13 \ll[\ldots]$ Leopardi, el lírico italiano de más fuerza de su tiempo, pesimista que vertió en sus obras el dolor físico de su vivir enfermizo y atormentado» (la traducción es mía), Ibidem.

\section{Sobre la autora}

Catedrática de Filología Italiana en la Universidad de Barcelona (España). Es autora de un centenar de artículos publicados en revistas especializadas, y capítulos de libro, así como varios libros sobre sus temas de investigación, que son la literatura italiana contemporánea, la traducción literaria y la recepción de la literatura italiana. Entre sus obras recientes mencionaremos La traducción y recepción de la literatura italiana e Italia en la prensa periódica durante el franquismo (ambos libros de 2014), así como La traducción en la creación del canon poético (Peter Lang, 2015). Es directora de la revista TRANSFER. 